\title{
A PREPARATORY STUDY OF THE BACILLUS ACID- OPHILUS IN REGARD TO ITS POSSIBLE THERAPEUTIC USE *
}

\author{
thomas MORgan Rotch, M.D., and ARTHUR I. Kendall, Ph.D. \\ BOSTON
}

The recent advances in our knowledge of the kiology of the gastroenteric tract have been so great that it seems time to begin a study of the practical use which can be made of individual organisms. Prominent among these organisms in the intestine is the obligate member of the acidoduric group, the Bacillus acidophilus.

It is to be noted in a general way that (1) this bacillus is a carbohydrate saprophyte; (2) it does not produce toxins; (3) it is a fermental organism, by fermental meaning an organism capable of breaking up the carbohydrates; (4) its habitat is the large intestine; (5) it thrives on carbohydrates, especially the sugars, such as dextrose, lactose and maltose; (6) it produces relatively large amounts of lactic acid from fermentable sugars; (7) it is antagonistic to the proteolytic organisms producing putrefaction, provided sugar is present; $(8)$ although it produces acid it grows best when the medium is less acid; (9) it appears to grow better below the ileocecal valve than above; (10) it is of interest clinically because, under suitable conditions, when the host is placed on a carbohydrate diet, the organism becomes more abundant and it inhibits and even replaces to a considerable extent the bacteria characteristic of a protein regimen (this replacement of the protein flora by acidoduric bacteria is accompanied by the gradual diminution and even disappearance in the urine of those substances which are the products of protein decomposition in the intestine); (11) the $B$. acidophilus is non-pathogenic for experimental animals and under normal conditions is found to be an inhabitant of the intestinal tract of man; (12) it has the ability to render that portion of the intestine where it is localized unfavorable to the development of foreign invading bacteria, and (13) it can form a considerable amount of acid from carbohydrates, forming this acid mostly below the ileocecal valve.

In this same acidoduric group the $B$. bulgaricus has somewhat different characteristics. Its habitat is chiefly above the ileocecal valve, so that the acid which it produces must be transported to the large intestine and must act at a distance from the chief site of putrefaction and is, therefore, probably less effective than if it were generated at the seat of infection.

\footnotetext{
*Read at the meeting of the American Pediatric Society, Lake Mohonk, N. Y., June, 1911.
} 
Its action is most pronounced above the ileocecal valve and grows less as it approaches the anus and the bacilli are only sparsely found in the feces.

In contradistinction to the acidoduric group of intestinal bacteria are the proteolytic bacteria and their characteristics are suggestive of the antagonism which exists between the two groups. (1) The putrefactive (proteolytic) organisms attack and thrive on the protein elements of the food; in certain instances some may become invasive. (2) They may develop toxins. (3) The end products of protein break-down of certain of these organisms are found in the urine and appear as aromatic bodies, these bodies acting as evidence of proteolytic intestinal activity and in some measure acting as indices of such activity. (4) The habitat of this group is said to be chiefly in the large intestine and they are consequently more likely to be abundant below the ileocecal valve. (5) They flourish and increase best in an alkaline or neutral medium. (6) They are inhibited by an acid medium especially by the presence of lactic acid. ( 7 ) Certain of these proteolytic bacteria are facultative and as a rule when protein and carbohydrate substances are present they attack the latter first and spare the former. (8) The addition to the diet of easily fermentable carbohydrates, together with restriction of protein food, is of material assistance in reducing the output of these putrefactive products. This results also in the diminution of the activity of the bacteria producing them.

There appears to be a considerable number of intestinal disturbances associated with the activities of putrefactive bacteria, some of which may be influenced by the activity of the $B$. acidophilus. As a well-known example of such putrefactive condition we might mention bacillary dysentery. There are three prominent bacilli which are associated with the clinical symptoms of bacillary dysentery: $B$. coli, streptococci and $B$. dysenteriax, one or both types. So far, however, as the clinical picture of bacillary dysentery is concerned a differential diagnosis between these organisms cannot be made. The actual recognition of which of these organisms is producing the disturbance is dependent on the bacteriologic examination. Hitherto bacillary dysentery has been regarded as a disease brought about solely by the action of the dysentery bacillus. Now, however, it appears in most cases, at least, that the three organisms mentioned above working symbiotically in appropriate intestinal conditions produce the symptom-complex of bacillary dysentery.

It is to be noted here that in those instances in which the acidoduric group of organisms has produced lactic acid in considerable amount (through the influence of a carbohydrate diet), $B$. coli, in virtue of its being a facultative organism, changes its metabolism from the proteolytic to the fermentative type. In consequence of this change in metabolism from the putrefactive to the fermentative type, this organism (B. coli), 
which on the protein regimen produced putrefactive products inimical to the patient, and which was, therefore, a symbiote of the dysentery bacillus, now becomes an antagonist to it by forming lactic acid.

In like manner, although we do not know so much about the antagonistic action in this connection, it has been observed that the disturbing streptococci are lessened in number apparently by the action of the lactic acid. It has also been shown in regard to $B$. dysenterice that certain changes in its action are brought about by the influence of the change to the carbohydrate type of diet. These changes, so far as is at present known, are as follows: the bacillus, whether of the Shiga or Flexner variety, is normally of the proteolytic type, and, having gained entrance to the intestinal tract, locates itself chiefly below the ileocecal valve. It begins its attack on the protein in the intestine and produces toxins. The bacilli may penetrate the mucosa and even the tissues as far as the mesenteric lymph-nodes and thus establish the bacillus as an infectious organism. The toxins are absorbed and especially affect the anterior horns of the spinal cord.

It is evident that any beneficial therapeutic action on the infectious organisms, so far as the action of the acidoduric group on them is concerned, is to diminish their numbers and to prevent their production of toxins by rendering the medium in which they are growing unsuited for their continued development, thus reducing the amount of toxin which they produce. The damage brought about by the toxins already absorbed cannot be undone. Early inhibitory action of the acid-producing bacteria is therefore indicated.

It has been noted in the changes in flora which take place in the intestine, when by any means the putrefactive types of organisms are gradually changed to the fermentative type, that during the process the bacterial flora first returns practically to a normal type for the nursling, namely, to $B$. bifidus mostly, and to $B$. acidophilus to a degree. Then, as the carbohydrate of the diet is increased the acidophilus increases in number and greater amounts of lactic acid are produced. Care, however, must be taken not to allow the acidophilus to flourish to such an extent as to establish an acid intolerance in the host.

To be mentioned as of especial significance in an investigation of this kind are certain known possibilities in connection with influences which may be brought to bear on the intestinal flora, and changes which may be accomplished by varying the quality of the food presented to intestinal organisms.

The character of the intestinal flora of man, both with respect to the types of organisms represented, and to the nature of the resulting chemical activities, depends largely on the composition of the diet of the host. A moment's reflection will convince one of the accuracy of this statement; the intestinal bacteria, to a very considerable extent, derive their suste- 
nance from the food in the alimentary canal, and as the food varies, so will the bacteria vary.

This variation in the nature of the bacteria comprising the intestinal fiora can conceivably change in two distinct directions, following alternations in the host's diet: either the organisms representative, for example, of a protein regimen may be replaced by an entirely new flora when the host changes his diet to one potentially carbohydrate, or the bacteria characteristic of the protein flora can shift their metabolism to meet the new conditions established by the carbohydrate regimen. As a matter of fact, both possibilities are realized, so that we may recognize three distinct classes of bacteria when the food of the host varies from a protein to a carbohydrate diet. We recognize first an obligate proteolytic group of bacteria, which thrive on a protein regimen. These organisms, best exemplified by $B$. putrificus, are in reality to be regarded as bacteria characteristic of putrefactive conditions in the intestinal tract. They disappear largely when the food is changed to carbohydrate.

The second group, the obligate fermentative bacteria, grow best and are most numerous when carbohydrate forms a prominent part of the diet. These bacteria in virtue of the considerable amounts of lactic acid which they form under suitable conditions, are antagonistic to, and tend to replace, the obligate putrefactive (proteolytic) bacteria. B. acidophilus is the best known of this group. The third group, the facultative organisms, are those which accommodate, within limits, their metabolism now to a protein, now to a carbohydrate regimen. They are usually present in either class of food but are more numerous on the protein type of regimen, because they are less affected by the products of putrefaction, than those of fermentation. $B$. coli and $B$. proteus are perhaps the best known of the facultative group.

We recognize, then, three well-defined types of bacterial activity in the intestinal tract, the putrefactive or proteolytic type, acting on the protein, the fermentative type acting by preference on carbohydrate, and the facultative type, which is by far the largest and best known, acting either on protein or carbohydrate. ${ }^{1}$

It is interesting and particularly important to recognize that the intestinal bacteria of normal nurslings are strictly fermentative. This is what one would expect, because the diet of the nursling is one well suited to encourage a fermentative flora, being composed of over 6 per cent. lactose and only about 1.5 per cent. protein. The reaction of a normal nursling's feces, furthermore, is strongly acid, due to the formation of lactic acid within the intestinal tract by this fermentative flora. It will be seen later on that this lactic acid, formed by the activity of

1. For a fuller discussion of these types, see Kendall, A. I. "Lactic Acid Bacteria: What They Are and What They Are Supposed to Do." Boston Med. and Surg. Jour., 1910, clxiii. 
fermentative organisms, is an important feature in keeping down putrefaction. In this connection it should be stated that the flora of normal nurslings contains few, if any, putrefactive organisms. As the child grows older and is placed on a cow's-milk diet, a change takes place in the intestinal flora: this change occurs less rapidly if the diet is strictly limited to modified milk, more rapidly if the diet is made up of unmodified milk or with the addition of protein. The change consists essentially in the replacement of the characteristic fermentative flora of the breastfed baby by greater or lesser numbers of putrefactive and facultative bacteria. It is marked by the beginning dominance of the facultative bacteria which tend to persist throughout life.

The cause underlying this change is not difficult to define: the change in flora is largely attributable to the relative decrease in carbohydrate, assciated with the increase of protein, two conditions favoring the development of a putrefactive or facultative flora.

It may be accepted as proved that the fermentative flora of the normal nursling is a valuable factor in protecting the host from invasion by foreign organisms, particularly of the proteolytic type. Consequently the lack of such a flora is to be regarded as a weakness in the normal defence against bacterial invasion of the intestinal tract. It has been shown for example ${ }^{2}$ that the intestinal flora in bacillary dysentery is essentially putrefactive in nature, at least in the more severe cases. It would appear logical and desirable, then, to attempt to reestablish and maintain the fermentative conditions at least in the intestinal tract of babies, and thus to encourage the reestablishment of the normal fermentative flora characteristic of such cases.

The question arises: How may this be accomplished? From what has been said it is evident that the increased administration of carbohydrate, and the restriction of protein will furnish the dietary conditions most favorable for the development of the appropriate organisms, and if the bacteria in question, the obligate fermentative bacteria, are present, and available in the intestinal canal, they may be brought up to their natural degree of activity. This process, however, takes a certain amount of time, and conditions may well arise in which the amount of time necessary to bring about this bacterial change will be prohibitive. For such cases, where time is a factor, and where it is desirable to populate the intestinal tract with useful types of fermentative organisms, the use of artificially prepared cultures of appropriate organisms is indicated, provided they can be obtained.

The observations presented in this paper are designed to show the feasibility of encouraging the growth of $B$. acidophilus by suitably modifying the food of the patient; if, however, acidophili are not present in

2. Kendall, A. I.: Bacillary Dysentery, Boston Med. and Surg. Jour., 1911, clxiv, 288. 
the alimentary tracts of patients, it is apparent that they cannot be encouraged to grow even by the most careful dietary regulation. In such cases we deem it advisable, from the theoretical point of view, to introduce cultures of acidophili, provided they have been obtained from sources known to be normal and absolutely free from objectionable conditions.

The characteristics of this organism ( $B$. acidophilus) have been described fully in a previous communication ${ }^{3}$ and need not be repeated here.

Cultures were readily obtained from two different patients by feeding maltose or lactose in moderate amounts. The details of the feeding experiments appear below.

The patients were first put on a diet in which protein was in moderate excess, and the characteristic protein flora dominated, as is shown by the protocols. As the diet was gradually changed to one in which carbohydrate predominated, there was a gradual restriction of the proteolytic flora, the more strictly proteolytic organisms diminished in numbers and the bacteria characteristic of the carbohydrate regimen, B. bifidus and $B$. acidophilus, gained the ascendency. These changes are of more than academic interest, for they indicate a possible method for controlling the proteolytic organisms, to a certain extent, at least, by dietary changes that are not only not harmful, but even under certain conditions beneficial to the host. While we are still quite in the dark concerning the absolute amount of importance to attribute to the presence of a more or less purely protein flora, the evidence is strongly in favor of the view that these organisms may not only be harmful themselves, but also that they may, and probably do, thrive in conditions suitable for the development of organisms like the typhoid or dysentery bacilli. On the other hand it is equally true that the conditions obtaining in the intestinal tract favorable for the continued development of the fermentative flora are inimical to the development of these pathogenic bacteria as well as for the putrefactive bacteria. Indeed, it appears to be a fact that the pathogenic organisms of the typhoid type, for example, are rather less resistant to the acids of the fermentative bacteria than are the more commonly metwith putrefactive bacteria which occur from time to time in the intestinal tract.

$I_{t}^{t}$ is not a matter of indifference which fermentative bacteria are encouraged to develop in the intestines; furthermore, certain organisms: appear to elect the small intestine, others the large intestine. As a wellknown example of the former, $B$. bulgaricus may be mentioned, while $B$. acidophilus is found most plentifully in the large intestine. Applyingthese principles clinically, it would appear that putrefactive conditions in the small intestine might be controlled more readily by an organism that

3. Kendall, A. I.: Jour. of Med. Research, 1910, N. S., xvii, 153. 
generated lactic acid in that portion of the alimentary canal, and $B$. bulgaricus would be selected for this purpose, while $B$. acidophilus theoretically, at least, would be more promising for putrefactions of the large intestine.

We have shown that $B$. acidophilus was increased in numbers on the sugar diet, and this method of encouraging the growth of this organism would appear to be relatively simple. The time necessary to bring this increase in $B$. acidophilus, however, varies considerably and doubtless depends on the numbers and activity of the organisms of this type remaining when the carbohydrate regimen is instituted. There are cases in which it would be desirable, or even essential, to be able to introduce large numbers of acidophili with the minimal expenditure of time, and we believe that artificial cultures of this organism are indicated. The problem arises: Is it possible to cultivate $B$. acidophilus in artificial media and still have it retain its desirable properties? Further studies on this point are needed before the question can be definitely decided.

It is evident from what has been stated that it is worth while to investigate whether the inhibitory action of $B$. acidophilus on certain harmful proteolytic organisms can be made use of therapeutically, and it is with this object in view that we have done some preparatory work on this subject. The first step in an inquiry of this kind is to obtain a strain of the acidophilus which has been shown to fulfil certain requirements, just as has been seen done with $B$. bulgaricus. This strain should be kept in pure culture ready for use according to the physician's demand.

After a series of investigations our interests centered on the following two cases which seemed to well illustrate how we obtained vigorous specimens of the $B$. acidophilus by carrying out suitable modifications of the diet according to the principles which we have described. These cases illustrate, furthermore, in a striking manner the alternations in the types of the fecal bacteria following the changes in diet. It is to be noted in studying the bacterial changes in these cases that $B$. acidophilus, like other bacteria, is selective of the kind of sugar that it prefers and thrives on, and that it is apparently encouraged to a greater degree of activity in a medium containing dextrose or maltose than any other sugars.

\section{CASE REPORTS}

CASE 1.-A boy, 6 months old, was being fed on a milk mixture containing a low protein, and for its sugar, lactose. The feces showed a moderate number of proteolytic organisms. On March 9 a high protein (3.50) was given and on March 13 the report of the feces was: Yellowish brown, with small curds; no mucus; slightly fetid odor. Bacteria: $B$. coli, $B$. subtilis, a few coccal forms; rather typical putrefactive feces both culturally and morphologically; practically no acidophili; many gelatin-liquefying forms. The food mixture was then changed to a low protein and the lactose replaced by a moderate amount of maltose-5 per cent. March 17 the report of the feces was: Yellow, with greenish color on the exposed parts; relatively homogeneous; odor not distinc- 
tive; $B$. coli and $B$. subtilis less in numbers; acidophili present in moderate numbers; a few bifid forms and a very small number of coccal forms appear; liquefying organisms greatly reduced in numbers and in activity. The maltose was then increased to 7 per cent. and the report on March 22 was: Yellow, granular and dry; $B$. coli and $B$. subtilis have almost disappeared; very few coecal forms persist; acidophilus and bifid forms increased. On March 27 the report was: Yellow, granular and relatively dry; bifid forms and acidophili present in considerable numbers; very few proteolytes; liquefying forms greatly diminished. On April 8 the report was: Small in amount, light color, with greenish color on exposed surfaces. Proteolytic bacteria have almost disappeared. Culturally, very few liquefying forms are left; they are very inactive; acidophilus and bifid forms still dominate.

In this case a markedly putrefactive intestinal flora was gradually changed to a practically normal nursling's, and then to one markedly acidoduric by providing at first a protein diet and then carbohydrate. The acidophili were vigorous and were separated and kept in pure culture. They were used to coagulate milk but did not make an entirely satisfactory buttermilk. The reason for this appears to have been that the culture obtained at this time was less vigorous than those usually obtained under suitable conditions. This is in line with the well-known variations which different strains of the same organism not infrequently exhibit in artificial media.

CASE 2,-A boy 17 months old, was being fed on a rather excessive protein diet and on March 13 a report of the feces was as follows: Greenish, homogeneous with a few small curds which were very hard; odor suggestive of putrefaction in intestinal tract (indol odor marked); culturally, $B$. coli few in numbers; many subtiloid organisms; a few coccal forms were present; liquefying forms numerous and active culturally; no acidophili found. The protein part of the diet was then decreased and on March 17 the report was: Light yellow, with slightly sour odor; good feces morphologically; $B$. coli still present in small numbers; $B$. subtilis present although in fewer numbers; liquefying colonies still active and numerous; practically no bifid forms or acidophili. The diet was then made largely carbohydrate and on March 22 the report was: Light brown, smooth, homogeneous; $B$. coli very few in numbers; $B$. subtilis greatly reduced, both numerically and in activity. Bifid forms appear in moderate numbers, together with acidophili; feces changing from the putrefactive to the fermentative type. On March 27 the report was: Brown, smooth, homogeneous; moderately voluminous; odor slightly sour; no starch demonstrable microscopically; culturally, very few $B$. coli, few $B$. subtilis, little liquefaction, and few liquefying forms; bifid forms in moderate numbers; considerable numbers of acidophilic bacteria; feces distinctly fermentative in type. On April 5 the report was: Smooth, brown, homogeneous; slightly acid reaction and sour odor; culturally, very few proteolytes were found and those that were recovered showed but little liquefying activity; bifid forms more numerous, acidophil dominant; typical fermentative feces.

In this case a markedly putrefactive intestinal condition was gradually changed under the influence of a carbohydrate regimen to a typical fermentative one. The acidophili were vigorous and were isolated and kept in pure culture. When introduced into milk the resulting lactic acid coagulation was exceedingly satisfactory and so this strain has been retained for further investigation. 
Successive transfers must now be made with this strain of the acidophilus to see if it retains its useful properties of antagonism to the putrefactive conditions, which it has been shown to possess, or whether when used in milk it becomes acclimated and loses these properties. It is probable that it retains its strong lactic acid forming properties as does the $B$. bulgaricus, but if it does not it is possible that it may still be given therapeutically in a carbohydrate menstruum. If it can be given in this way it seems reasonable to suppose that the acidophilus will be superior to the bulgaricus in its power of inhibiting those putrefactive disturbances which are located below the ileocecal valve. It is here to be especially noted that while the activity of the bulgaricus grows less when it passes the ileocecal valve, that of the acidophilus increases, the latter always approaching in its downward descent its home, where it flourishes best, increases in number and vigor and produces most acid.

197 Commonwealth Avenue. 